\title{
Antibiotic Resistance and Virulence Profiles of Klebsiella pneumoniae Strains Isolated From Different Clinical Sources
}

\section{OPEN ACCESS}

Edited by:

Naofumi Naga,

Shibaura Institute of Technology,

Japan

Reviewed by:

Anh K. Lam,

Indiana University, United States

Govind Vediyappan,

Kansas State University, United States

*Correspondence:

Sara Soto

sara.soto@isglobal.org

Specialty section:

This article was submitted to Molecular Bacterial Pathogenesis,

a section of the journal

Frontiers in Cellular and

Infection Microbiology

Received: 08 July 2021

Accepted: 16 August 2021

Published: 01 September 2021

Citation:

Ballén V, Gabasa Y, Ratia C, Ortega R, Tejero M and Soto S (2021) Antibiotic

Resistance and Virulence Profiles of Klebsiella pneumoniae Strains Isolated From Different Clinical Sources. Front. Cell. Infect. Microbiol. 11:738223.

doi: 10.3389/fcimb.2021.738223

\author{
Victoria Ballén, Yaiza Gabasa, Carlos Ratia, Raquel Ortega, Marc Tejero and Sara Soto*
}

ISGlobal, Hospital Clínic-Universitat de Barcelona, Barcelona, Spain

Klebsiella pneumoniae is a Gram-negative bacterium capable of colonizing, invading, and causing infections in different anatomical sites of the human body. Its ability to evade the immune system, its increasing antimicrobial resistance and the emergence of hypervirulent pathotypes have become a major challenge in the medical field. In this study, 127 strains from different clinical sources (urine, respiratory tract or blood) were characterized for antimicrobial resistance, the presence of virulence factor genes, serum resistance, hypermucoviscosity and the ability to form biofilms. Specific characteristics of the uropathogenic strains were examined and compared with the other clinical groups.

Differences were found between urine and the other groups of strains. Urine strains showed the highest antibiotic resistance (64.91\%) compared to blood (63.64\%) or respiratory strains (51.35\%) as well as the highest extended-spectrum beta-lactamases (ESBL) production. These strains also showed statistically significant high resistance to fosfomycin $(24.56 \%)$ compared to the other groups ( $p=0.008$ ). Regarding virulence, $84.21 \%$ of the urine strains presented the uge gene, showing a statistically significant difference ( $p=0.03$ ) compared to the other clinical sources, indicating a possible role of this gene in the development of urinary tract infection. In addition, $46 \%$ of biofilm-forming strains belonged to the urine sample group $(p=0.043)$. In conclusion, $K$. pneumoniae strains isolated from urine samples showed higher antimicrobial resistance, ESBL production, and biofilm-forming ability compared to those isolated from respiratory or blood samples. The rapid spread of clinical strains with these characteristics is of concern, and new therapeutic alternatives are essential to mitigate their harmful effects.

Keywords: Klebsiella pneumoniae, antimicrobial resistance, virulence, biofilm, urinary tract infections 


\section{INTRODUCTION}

Klebsiella pneumoniae is an opportunistic pathogen associated with a variety of infections, being urinary tract infections (UTIs) among the most common worldwide. K. pneumoniae is considered an important uropathogen in ambulatory patients (Foxman, 2010). Although UTIs do not result in a high mortality rate, they represent a significant economic burden to the health care system as they increase treatment costs.

However, $K$. pneumoniae is not only responsible for UTIs but also for respiratory and bloodstream infections (Podschun and Ullmann, 1998). It is one of the species recognized as part of the ESKAPE group, associated by their characteristic potential to escape or evade the action of antimicrobial agents. This acronym comprises six highly virulent and antibiotic-resistant bacterial pathogens including Enterococcus faecium, Staphylococcus aureus, $K$. pneumoniae, Acinetobacter baumannii, Pseudomonas aeruginosa, and Enterobacter species. Additionally, the World Health Organization (WHO) lists K. pneumoniae as one of the species of high priority and promotes the research and development of new antibiotics due to the growing global problem of antimicrobial resistance (World Health Organization, 2017).

Uropathogenic bacteria have some characteristics that favor the colonization of human cells, such as the production of adhesins, siderophores, and toxins (Foxman, 2010). In the same way, these bacteria can adhere to medical devices forming biofilms, avoiding the immune system and favoring the antimicrobial therapy failure (Dybowska-Sarapuk et al., 2017). For this reason, the characterization of $K$. pneumoniae antimicrobial resistance, its ability to form biofilm and its virulence are key to understanding the pathogenicity of this bacteria in the clinical setting and to achieve more efficient antimicrobial therapy.

The aim of this study was to characterize a collection of $K$. pneumoniae strains isolated from different clinical sources (urine, respiratory tract, or blood) in terms of antimicrobial resistance, the presence of virulence factor genes, serum resistance, hypermucoviscosity, and the ability to form biofilms. The specific characteristics of the uropathogenic strains were studied and compared to K. pneumoniae strains isolated from respiratory and/or blood samples.

\section{MATERIALS AND METHODS}

\section{Bacterial Strains}

One hundred twenty-seven K. pneumoniae clinical strains from four Catalonian hospitals (Hospital Clinic de Barcelona, Hospital Universitario de Bellvitge, Hospital del Mar, and Hospital Universitario Mutua de Terrassa) were collected over six months from 2016 to 2017. Among these, 57 strains were obtained from urine (1 urinary catheter, 1 suprapubic aspiration, and 55 midstream urine), 37 from respiratory samples (7 sputum, 10 bronchoalveolar aspirates, 4 tracheal samples, 1 bronchoalveolar lavage, 15 non-classified respiratory samples), and 33 strains were obtained from blood. Strains were identified as K. pneumoniae using matrix-assisted laser desorption ionization-time-of-flight mass spectrometry (MALDI-TOF/MS) (Bruker Daltonik GmbH, Bremen, Germany). The modified score values suggested by the manufacturer were used: A score $\geq 2.3$ meant species identification; a score between 2.0 and 2.299 meant genus identification and probable species identification; a score between 1.7 and 1.9 meant probable genus identification; and a score $<1.69$ meant non-reliable identification. Strains with a score $\geq 2.3$ classified as $K$. pneumoniae were included in the study. The strains were stored in skim milk (BD) at $-80^{\circ} \mathrm{C}$. All data on the strains used in this study can be found in the Supplementary Material.

\section{Determination of Antimicrobial Resistance}

Determination of antimicrobial resistance was assessed by disk diffusion or broth microdilution (in the case of colistin) methods following the Clinical and Laboratory Standards Institute guidelines (CLSI, 2020). The antimicrobial agents tested by Kirby-Bauer method were amoxicillin/clavulanate $(20 / 10 \mu \mathrm{g})$, aztreonam $(30 \mu \mathrm{g})$, cefepime $(30 \mu \mathrm{g})$, ceftazidime $(30 \mu \mathrm{g})$, chloramphenicol $(30 \mu \mathrm{g})$, ciprofloxacin $(5 \mu \mathrm{g})$, fosfomycin $(200$ $\mu \mathrm{g} / 50 \mu \mathrm{g}$ of glucose-6-phosphate), gentamicin $(10 \mu \mathrm{g})$, imipenem $(10 \mu \mathrm{g})$, piperacillin/tazobactam $(100 / 10 \mu \mathrm{g})$, and trimethoprimsulfamethoxazole $(1.25 / 23.75 \mu \mathrm{g})$. E. coli ATCC 25922 was used as quality control.

Strains were classified according to their antimicrobial resistance profile as susceptible, resistant to 1 or 2 antimicrobial categories, multidrug-resistant (MDR), extensively drug-resistant (XDR) or pandrug-resistant (PDR). The European Centre for Disease Prevention and Control (ECDC) proposed the MDR, XDR, and PDR definitions. An isolate is considered MDR if it is nonsusceptible to at least 1 agent in $\geq 3$ antimicrobial categories, defined as XDR if the isolate is non-susceptible to at least 1 agent in all but 2 or fewer antimicrobial categories, and PDR if the isolate is non-susceptible to all listed antimicrobial agents (Magiorakos et al., 2012).

Extended-spectrum beta-lactamases (ESBL) production was evaluated by the ESBL test and carbapenemase production was analyzed by modified carbapenem inactivation and EDTAmodified carbapenem inactivation methods following CLSI guidelines (CLSI, 2020).

\section{String Test}

The string test was performed to identify the hypermucoviscous (HMV) phenotype. The strains were grown in BD Columbia agar with $5 \%$ sheep blood (Beckton Dickinson) at $37^{\circ} \mathrm{C}$ overnight. Afterwards, a bacteriological inoculation loop was used to stretch a mucous bacterial colony. The HMV phenotype was positive when a string $>5 \mathrm{~mm}$ in length was observed (Fang et al., 2004).

\section{Determination of Virulence and Antimicrobial Resistance Genes}

A single colony from an overnight culture plate was selected and suspended in $100 \mu \mathrm{L}$ of sterilized Milli-Q water. The suspension was boiled for $10 \mathrm{~min}$ and centrifuged for $10 \mathrm{~min}$ at $13000 \mathrm{rpm}$. The supernatant was used as the DNA template.

Polymerase chain reaction (PCR) was performed to detect different virulence genes: fimbriae ( $f i m D, f i m H, m r k C, m r k D)$, 
capsule-associated genes ( $y c f M, w a b G$, uge and $r m p A)$, siderophores (entB, iucA, irp2, ybtS, fyuA, iroN), colibactin genes $(c l b A, c l b Q)$, and capsular serotypes (K1, K2).

ESBL-associated genes $b l a_{\mathrm{SHV}}, b l a_{\mathrm{TEM}}, b l a_{\mathrm{CTX}-\mathrm{M}-1}, b l a_{\mathrm{OXA} 48}$, in addition to genes conferring resistance to quinolones [aac (6)$I b-c r, q n r B]$, aminoglycosides $(a a d b)$, and sulfonamides (sul1 and sul2), were detected by PCR. The primers used are listed in Table 1.

\section{Biofilm Formation and Quantification}

Biofilm formation was performed using the protocol described by O’Toole and Kolter, (1998) with some modifications. Briefly,

TABLE 1 | Primers to detect virulence and antimicrobial resistance genes.

\begin{tabular}{|c|c|c|c|c|}
\hline Target gene & $\begin{array}{l}\text { Primer sequence } \\
\qquad\left(5^{\prime} \rightarrow 3^{\prime}\right)\end{array}$ & $\begin{array}{l}\text { Melting temperature } \\
\left(\operatorname{Tm}^{\circ} \mathrm{C}\right)\end{array}$ & Size of amplicon (bp) & Reference \\
\hline$f i m D-F$ & GTTACGCCTATCTGAATCTACAGAG & 57 & 1114 & This study \\
\hline $\operatorname{fim} D-\mathrm{R}$ & GACCAGTTGATATCGTCCACG & & & \\
\hline$f i m H-F$ & GAAAAAAATAATCCCССTGTTCAC & 57 & 855 & This study \\
\hline $\operatorname{fimH}-\mathrm{R}$ & GTAACCTGGCCTGTGGTC & & & \\
\hline$m r k C-F$ & GCTCAACTCCATCGTCAGC & 57 & 1056 & This study \\
\hline$m r k C-\mathrm{R}$ & CGCGAGTTATTGAACGAGGTG & & & \\
\hline$m r k D-F$ & CGCTIITATCGTCTTAATG & 55 & 880 & This study \\
\hline$m r k D-\mathrm{R}$ & GTGATGTAGCGGGTCTCCTG & & & \\
\hline$y c f M-F$ & ATCAGCAGTCGGGTCAGC & 57 & 160 & (Candan and Aksöz, 2015) \\
\hline$y c f M-R$ & CTTCTCCAGCATTCAGCG & & & \\
\hline entB - F & АTाССTCAACTTCTGGGGC & 60 & 371 & (Candan and Aksöz, 2015) \\
\hline entB - R & AGCATCGGTGGCGGTGGTCA & & & \\
\hline iucA - F & AATCAATGGCTATTCCCGCTG & 58 & 239 & (Garza-Ramos et al., 2018) \\
\hline iucA - R & CGCTTCACTTCTICACTGACAGG & & & \\
\hline irp2 - F & GCTACAATGGGACAGCAACGAC & 58 & 230 & (Garza-Ramos et al., 2018) \\
\hline irp2 - R & GCAGAGCGATACGGAAAATGC & & & \\
\hline$y b t S-F$ & GACGGAAACAGCACGGTAAA & 53 & 242 & (Compain et al., 2014) \\
\hline$y b t S-R$ & GAGCATAATAAGGCGAAAGA & & & \\
\hline fуuA - F & TTCCACCAACACCATCCAG & 58 & 817 & This study \\
\hline fyuA - R & CAGGTCAGGTCACTGTATGC & & & \\
\hline $\operatorname{iroN}-\mathrm{F}$ & AACCGCCAGATCGATATTCG & 58 & 827 & This study \\
\hline iroN - R & TTAATCTCACCGCTGGTTCG & & & \\
\hline$r m p A-F$ & CATAAGAGTATTGGTTGACAG & 56 & 461 & (Compain et al., 2014) \\
\hline$r m p A-\mathrm{R}$ & CTTGCATGAGCCATCTTCA & & & \\
\hline wabG - F & CGGACTGGCAGATCCATATC & 58 & 683 & (Jian-li et al., 2017) \\
\hline$w a b G-R$ & ACCATCGGCCATTTGATAGA & & & \\
\hline uge - F & GATCATCCGGTCTCCCTGTA & 59 & 535 & This study \\
\hline uge - R & TCTTCACGCCTTCCTTCACT & & & \\
\hline$K 1-F$ & GGTGCTCTITACATCATTGC & 59 & 1283 & (Lin et al., 2014) \\
\hline$K 1-\mathrm{R}$ & GCAATGGCCATITGCGTTAG & & & \\
\hline$K 2-F$ & GACCCGATATTCATACTTGACAGAG & 59 & 641 & (Lin et al., 2014) \\
\hline$K 2-\mathrm{R}$ & CCTGAAGTAAAATCGTAAATAGATGGC & & & \\
\hline$c / b A-F$ & CTAGATTATCCGTGGCGATTC & 51 & 1002 & (Morgan et al., 2019) \\
\hline$c / b A-R$ & CAGATACACAGATACCATTCA & & & \\
\hline$c l b Q-F$ & CTTGTATAGTTACACAACTATIT & 50 & 821 & (Morgan et al., 2019) \\
\hline$c / b Q-R$ & TTATCCTGTTAGCTTCGTTC & & & \\
\hline blaSHV-1 - F & ATGCGTTATATTCGCCTGTG & 49 & 747 & (Wiegand et al., 2007) \\
\hline blaSHV-1 - R & TGCTITGTTATTCGGGCCAA & & & \\
\hline blaTEM-1 - F & TCGCCGCATACACTATTCTCAGAATGA & 53 & 445 & (Monstein et al., 2007) \\
\hline blaTEM-1 - R & ACGCTCACCGGCTCCAGATTTAT & & & \\
\hline blaCTX-M1 - F & AAAAATCACTGCGCCAGTTC & 52 & 415 & (Woodford et al., 2006) \\
\hline blaCTX-M1 - R & AGCTTATTCATCGCCACGTT & & & \\
\hline blaOXA48 - F & TTGGTGGCATCGATTATCGG & 57 & 744 & (Queenan and Bush, 2007) \\
\hline blaOXA48 - R & GAGCACTTCTITTGTGATGGC & & & \\
\hline$a a c\left(6^{\prime}\right)-I b-c r-F$ & TTGCGATGCTCTATGAGTGGCTA & 60 & 482 & (Park et al., 2006) \\
\hline$a a c\left(6^{\prime}\right)-1 b-c r-R$ & CTCGAATGCCTGGCGTGTTT & & & \\
\hline$q n r B-F$ & GATCGTGAAAGCCAGAAAGG & 52 & 469 & (Robicsek et al., 2006) \\
\hline$q n r B-R$ & ACGATGCCTGGTAGTTGTCC & & & \\
\hline$a a d b-F$ & ATGGACACAACGCAGGTCGC & 55 & 534 & (Guo et al., 2016) \\
\hline$a a d b-R$ & TTAGGCCGCATATCGCGACC & & & \\
\hline sul1 - F & CTTCGATGAGAGCCGGCGGC & 63 & 436 & (Guerra et al., 2004) \\
\hline sul1 - R & GCAAGGCGGAAACCCGCGCC & & & \\
\hline sul2 - F & TCAACATAACCTCGGACAGT & 55 & 707 & (Guerra et al., 2004) \\
\hline sul2 - R & GATGAAGTCAGCTCCACCT & & & \\
\hline
\end{tabular}


isolates were cultured in Luria Bertani (LB) agar (Miller's LB AGAR, Condalab) for $24 \mathrm{~h}$ at $37^{\circ} \mathrm{C}$. Then, a single colony of each strain was grown in $10 \mathrm{~mL}$ of LB broth (LB, Condalab) for $24 \mathrm{~h}$ at $37^{\circ} \mathrm{C}$ with shaking at $180 \mathrm{rpm}$. Afterwards, overnight cultures were diluted 1:100 in fresh medium supplemented with $0.25 \%$ glucose, $200 \mu \mathrm{L}$ were inoculated in polystyrene microtiter plates (Nunc $^{\mathrm{TM}}$ Edge 2.0 96-well plate, non-treated, with lid, VWR International), and incubated for $48 \mathrm{~h}$ at $37^{\circ} \mathrm{C}$ in static conditions. Culture medium without inoculum was used as a sterility control.

After incubation, the liquid of each well was removed, washed once with $1 \mathrm{x}$ phosphate-buffered saline (PBS), and dried at $65^{\circ} \mathrm{C}$. Plates were then stained with crystal violet (CV) (2\% v/v) for $10 \mathrm{~min}$ at room temperature. Next, plates were washed once with 1x PBS and fixed at $65^{\circ} \mathrm{C}$ for $60 \mathrm{~min}$. The $\mathrm{CV}$ was detached by the addition of $33 \%$ glacial acetic acid and biomass was calculated measuring the optical density (OD) at $580 \mathrm{~nm}$ using a microplate reader (EPOCH 2 microplate reader; BioTek, VT). The experiment was carried out in three technical and biological replicates.

The strains were classified according to the criteria of Stepanović et al. (2007) as: non-biofilm formers (OD $\leq 0.150$ ), weak biofilm formers $(\geq 0.151$ OD $\leq 0.300)$, moderate biofilm formers $(\geq 0.301 \mathrm{OD} \leq 0.60)$, or strong biofilm formers (OD $\geq 0.601)$.

\section{Serum Resistance}

Serum resistance was analyzed following the procedure described by (Podschun et al., 2016). Briefly, $25 \mu \mathrm{L}$ of inoculum of $2.5 \times 10^{6}$ colony forming units $(\mathrm{CFU}) / \mathrm{mL}$ of a mid-log phase culture were mixed with $75 \mu \mathrm{L}$ of normal human serum obtained from healthy volunteers in 96-well polystyrene, round-bottomed microtiter plates (Greiner bio-one). The mixture was incubated at $37^{\circ} \mathrm{C}$ for 1,2 , and $3 \mathrm{~h}$. After incubation, the cell count was determined using 10-fold serial dilutions and conventional plating in LB agar (Miller's LB AGAR, Condalab). The test was graded as shown in Table 2. The assay was performed in triplicate.

\section{Statistical Analysis}

Statistical analysis was performed using the Chi-square test with the IBM SPSS Statistics software for Windows, version 21.0. $P$-values $<0.05$ were considered statistically significant.

\section{RESULTS}

\section{Antimicrobial Resistance}

Overall, 50 isolates (39.37\%) were susceptible to all the antimicrobial agents tested, 24 isolates (18.90\%) were non-susceptible to 1 or 2 categories of antibiotics, 51 isolates $(40.16 \%)$ were considered multidrug-resistant (MDR), and 2 strains (1.57\%) were considered extensively drug-resistant (XDR). None was classified as pandrug-resistant (Figure 1). The percentages of resistance for each antibiotic tested are shown in Figure 2.

Among uropathogenic K. pneumoniae, we found 20 susceptible strains (35.09\%), 12 strains $(21.05 \%)$ were nonsusceptible to 1 or 2 categories, and 25 were MDR strains
TABLE 2 | Classification of serum resistance.

\begin{tabular}{lccc}
\hline & \multicolumn{2}{c}{ Cell counts/mL (compared to the original inoculum) } \\
\cline { 2 - 4 } & $\mathbf{1} \mathbf{h}$ & $\mathbf{2} \mathbf{h}$ & $\mathbf{3} \mathbf{h}$ \\
\hline Grade 1 & $<10 \%$ & $<10 \%$ & $<0.1 \%$ \\
Grade 2 & $10 \%-100 \%$ & $10 \%-100 \%$ & $<10 \%$ \\
Grade 3 & $>100 \%$ & $<100 \%$ & $<100 \%$ \\
Grade 4 & $>100 \%$ & $>100 \%$ & $<100 \%$ \\
Grade 5 & $>100 \%$ & $>100 \%$ & $>100 \%$ (decreasing) \\
Grade 6 & $>100 \%$ & $>100 \%$ & $>100 \%$ (increasing) \\
\hline
\end{tabular}

(43.86\%). The uropathogenic group showed the highest overall antibiotic resistance $(64.91 \%)$ compared to blood (63.64\%) and respiratory tract $(51.35 \%)$ strains, although these differences were not statistically significant. The highest percentages of antimicrobial resistance were observed for ciprofloxacin (42.11\%), amoxicillin/clavulanic acid (36.84\%), trimethoprimsulfamethoxazole $(36.84 \%)$ and ceftazidime $(33.33 \%)$. Uropathogenic $K$. pneumoniae also showed the highest resistance to fosfomycin $(24.56 \%)$ with the difference being statistically significant $(\mathrm{p}=0.008)$ compared to the other groups.

Among the respiratory group, 18 strains (48.65\%) were classified as susceptible, 6 strains $(16.22 \%)$ as non-susceptible to 1 or 2 categories, 11 strains $(29.73 \%)$ as MDR, and 2 strains $(5.40 \%)$ as XDR. The highest percentages of antimicrobial resistance were observed with amoxicillin/clavulanic acid (40.54\%), ciprofloxacin (37.84\%), piperacillin/tazobactam (37.84\%) and ceftazidime (35.14\%). In comparison to the other groups, respiratory strains showed the highest resistance to imipenem (24.32\%) and piperacillin/tazobactam (37.84\%), being these differences statistically significant ( $\mathrm{p}=0.003$ both).

Strains isolated from blood samples were classified as follows: 12 strains (36.36\%) were susceptible, 6 strains (18.18\%) were non-susceptible to 1 or 2 categories, and 15 strains (45.46\%) were MDR. The highest percentages of antimicrobial resistance were observed with ceftazidime $(45.45 \%)$, followed by ciprofloxacin, amoxicillin/clavulanic acid and trimethoprimsulfamethoxazole with a percentage of resistance of $42.42 \%$ each.

\section{Presence of Antimicrobial Resistance Genes}

Fifty-five isolates (43.31\%) were ESBL producers. Among these, $24(43.6 \%)$ were isolated from urine, $16(29.1 \%)$ from blood, and $15(27.3 \%)$ from the respiratory tract. The gene most frequently detected was $b l a_{\mathrm{SHV}-1}(85.45 \%)$, followed by $b l a_{\mathrm{CTX}-\mathrm{M} 1}(78.95 \%)$, and $b l a_{\text {TEM-1 }}(71.93 \%)$. Thirteen strains $(10.24 \%)$ were carbapenemase producers: 9 from the respiratory tract (69.2\%), 4 from blood (30.8\%) and none from urine. Among these, 10 $(76.92 \%)$ harbored the $b l a_{\text {OXA48 }}$ gene. Concerning the 52 (40.94\%) strains resistant to ciprofloxacin, 20 (38.46\%) harbored the $a a c\left(6^{\prime}\right)-I b-c r$ gene, and 27 (51.92\%) harbored the $q n r B$ gene. One strain among the group of gentamicin-resistant strains harbored the $a a d b$ gene. Regarding the 44 strains classified as resistant to trimethoprim-sulfamethoxazole (34.65\%), six (13.64\%) and $29(65.91 \%)$ carried the sull and the sul2 genes, respectively. 


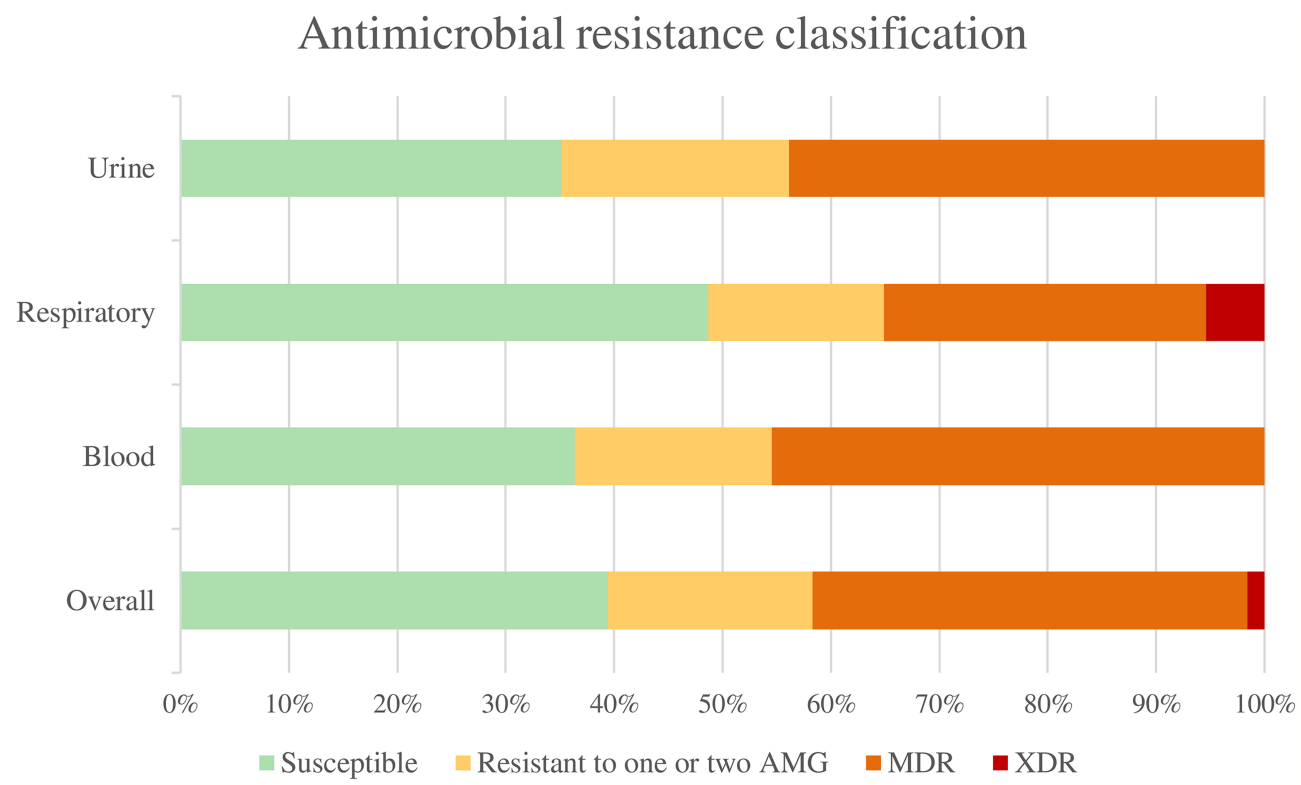

FIGURE 1 | Antimicrobial resistance classification. AMG, Antimicrobial groups; MDR, multidrug-resistant; XDR, extensively drug-resistant.

Overall antimicrobial resistance profiles

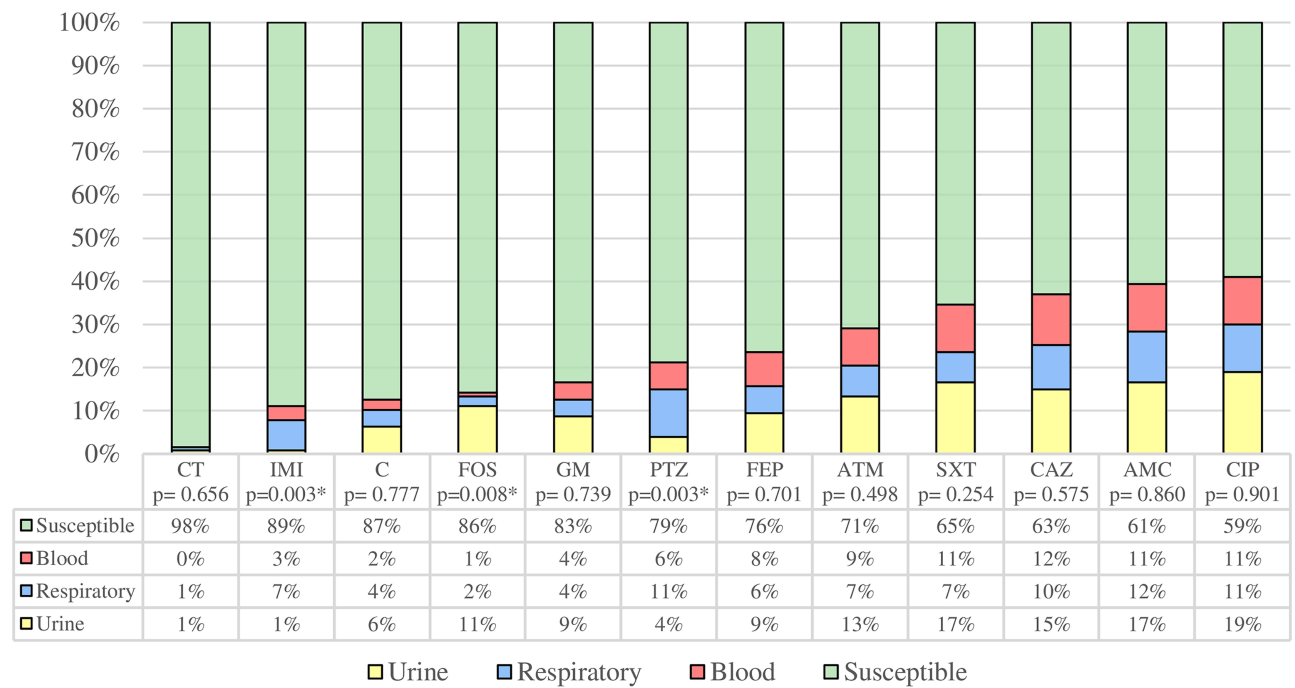

FIGURE 2 | Overall antimicrobial resistance profiles were categorized by clinical source. Susceptibility rate includes all clinical sources, while resistance rates are classified by urine, respiratory tract, and blood. * Statistically significant ( $\mathrm{p}$ < 0.05). CT, colistin; IMI, imipenem; C, chloramphenicol; FOS, fosfomycin; GM, gentamicin; PTZ, piperacillin/tazobactam; FEP, cefepime; ATM, aztreonam; SXT, trimethoprim-sulfamethoxazole; CAZ, ceftazidime; AMC, amoxicillin/clavulanate; CIP, ciprofloxacin.

\section{Presence of Virulence Genes}

The results obtained are shown in Figure 3. All the isolates carried the $y c f M$, ent $B$, and $w a b G$ genes. The fimD, fimH, $m r k C$, and $m r k D$ genes were almost ubiquitous among the strains (98.43\%). The prevalence of the other virulence genes was as follows: uge (73.23\%), irp2 (41.73\%), ybtS (40.94\%), fyuA (40.16\%), iucA (11.02\%), rmpA (7.09\%), $\operatorname{iroN}(5.51 \%), c l b A(1.57 \%)$, and $c l b Q(1.57 \%)$.
Among all these genes, the uge gene showed a higher prevalence among urinary strains compared to respiratory and blood samples, being the difference statistically significant ( $\mathrm{p}=$ 0.033). On the other hand, the iucA, ybtS, fyuA and rmpA genes were statistically more prevalent in respiratory isolates than urinary or blood strains $(\mathrm{p}=0.001, \mathrm{p}=0.022, \mathrm{p}=0.017$, and $\mathrm{p}=0.002$, respectively). 


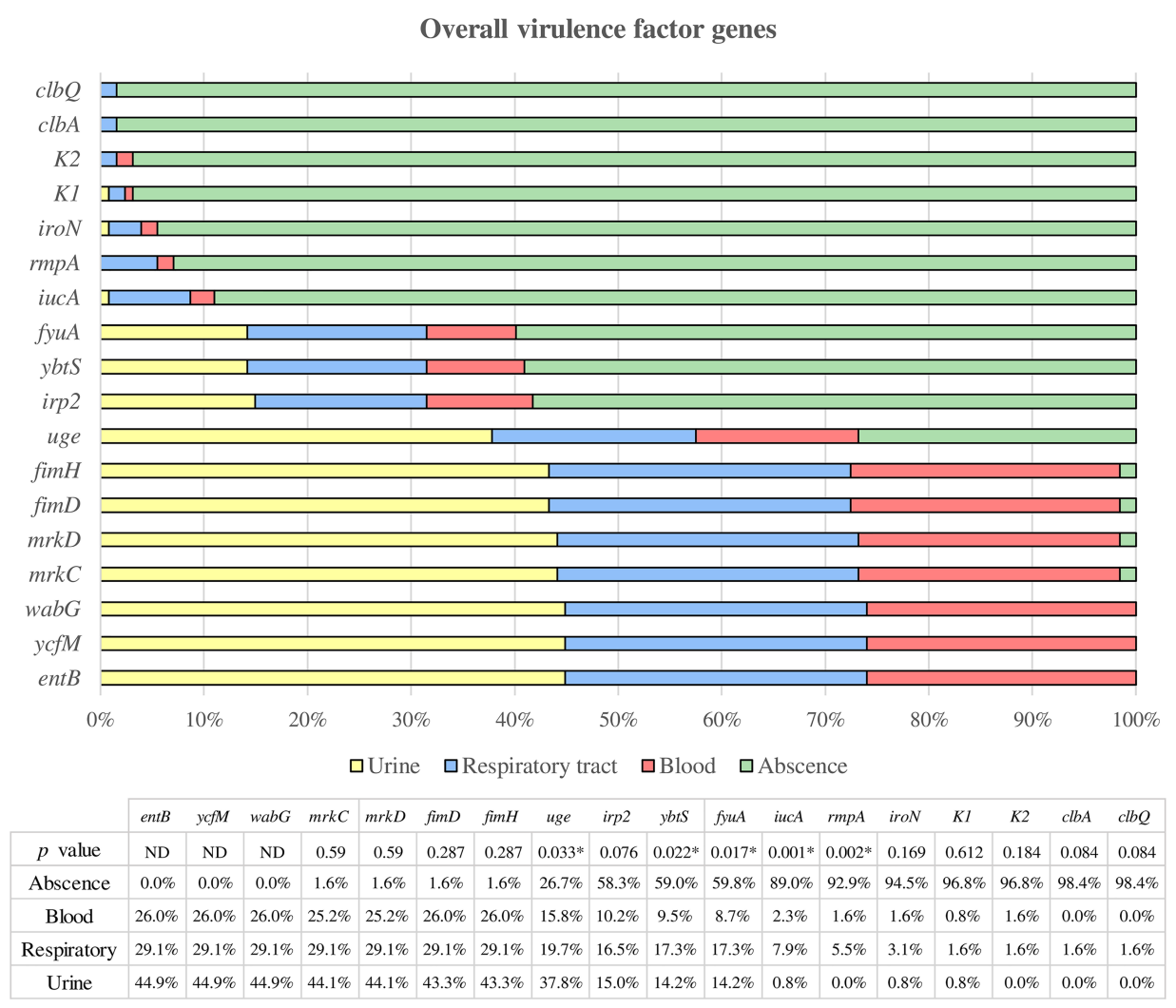

FIGURE 3 | Overall virulence factor genes were categorized according to the clinical source. All strains in which the respective gene is not present are assigned to the "absence" category, while the presence of the gene is classified according to the clinical source (urine, respiratory and blood). ${ }^{*}$ Statistically significant $(p<0.05)$. ND, Not determined.

\section{String Test and Serum Resistance}

In the present study, 17 K. pneumoniae strains (13.39\%) were identified as hypermucoviscous by the string test. Among these, 7 strains were isolated from urine, 7 from the respiratory tract and 3 from blood.

Table 3 shows the results of serum resistance. We observed that more than $50 \%$ of the strains tested were highly resistant to serum, but there was no correlation between this test and the other characteristics under study (type of isolate, biofilm formation, hypermucoviscosity, antimicrobial resistance classification, or the presence of virulence genes).

\section{Biofilm Formation and Quantification}

Twenty-five strains (19.69\%) were classified as non-biofilm formers, $35(27.55 \%)$ as weak biofilm formers, $42(33.07 \%)$ as moderate biofilm formers, and 25 strains (19.69\%) as strong biofilm formers. Among the 102 biofilm-forming strains, including weak, moderate and strong, 47 (46.1\%) were from urine, 30 (29.4\%) were from blood, and 25 strains $(24.5 \%)$ were from the respiratory tract (Figure 4). When grouping the strains into biofilm-forming and non-biofilm-forming, we found that the highest number of biofilmforming strains were from urine, and compared to the other groups of isolates, the difference was statistically significant $(p=0.043)$.

TABLE 3 | Results of serum resistance test.

\begin{tabular}{|c|c|c|c|c|}
\hline & \multicolumn{4}{|c|}{ Serum resistance } \\
\hline & 57 (44.88\%) & $37(29.13 \%)$ & 33 (25.99\%) & 127 (100\%) \\
\hline Grade 2 & $6(10.53 \%)$ & $6(16.22 \%)$ & $3(9.09 \%)$ & $15(11.81 \%)$ \\
\hline Grade 3 & $3(5.26 \%)$ & $1(2.70 \%)$ & $0(0 \%)$ & $4(3.15 \%)$ \\
\hline Grade 4 & $1(1.75 \%)$ & $0(0 \%)$ & $0(0 \%)$ & 1 (0.79\%) \\
\hline
\end{tabular}


A

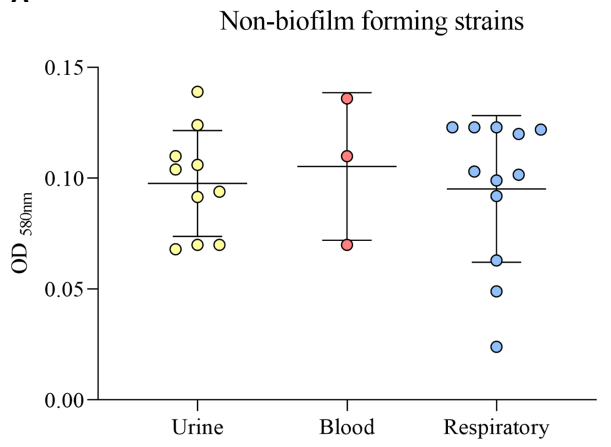

B

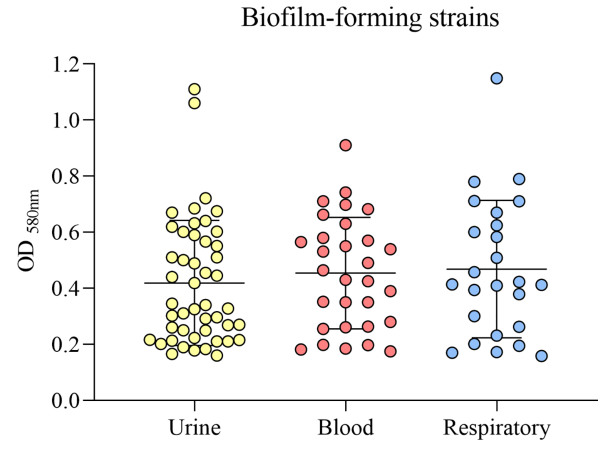

FIGURE 4 | Classification of biofilm formation ability by the clinical source (urine, blood, or respiratory tract). (A) Non-biofilm-forming strains. (B) Biofilm-forming strains.

\section{Relationship Between Antimicrobial Resistance and Virulence}

There was a statistically significant relationship between the strains carrying the $i u c A, \operatorname{rmp} A$ and uge genes and the strains classified as susceptible in the antimicrobial resistance classification ( $\mathrm{p}=0.028 ; \mathrm{p}=0.006 ; \mathrm{p}<0.001)$.

Excluding colistin, we observed a statistically significant relationship between the presence of the uge gene and the susceptibility to the other antibiotics tested ( $\mathrm{p}<0.005)$. Likewise, strains harboring the $r m p A$ gene were more susceptible to amoxicillin/clavulanate, ciprofloxacin, trimethoprim-sulfamethoxazole and cefepime $(\mathrm{p}=0.012 ; \mathrm{p}=$ $0.010 ; \mathrm{p}=0.023 ; \mathrm{p}=0.046)$. Strains harboring iroN or iucA genes were more susceptible to amoxicillin/clavulanate $(\mathrm{p}=0.028 ; \mathrm{p}=$ $0.042)$ and ciprofloxacin ( $p=0.023 ; \mathrm{p}=0.032)$. In addition, strains carrying iroN genes were also more susceptible to trimethoprim-sulfamethoxazole $(\mathrm{p}=0.048)$.

A similar relationship was found between the strains carrying the irp2 gene and susceptibility to ceftazidime and gentamicin $(\mathrm{p}=0.020 ; \mathrm{p}=0.040)$, and the strains harboring the $y b t S$ gene and the susceptibility to gentamicin $(\mathrm{p}=0.009)$.

The irp2, ybtS, and $f y u A$ genes showed a higher prevalence among ESBL-producing strains $(\mathrm{p}<0.001, \mathrm{p}=0.02$, and $\mathrm{p}=$ 0.012 , respectively), and the $\operatorname{rmpA}$ and the uge genes were more prevalent among the non-ESBL-producing strains ( $\mathrm{p}=$ 0.043 and $\mathrm{p}<0.001$, respectively). Nevertheless, the irp2, ybtS and uge genes showed a higher prevalence among noncarbapenemase-producer strains $(\mathrm{p}=0.034 ; \mathrm{p}=0.029$; $\mathrm{p}=0.003)$.

Concerning the hypermucoviscous phenotype, the lack of the iucA, iroN, and $\operatorname{rmpA}$ genes was related to the nonhypermucoviscous phenotype ( $\mathrm{p}<0.05$ in all cases). Hypermucoviscous strains also showed a lower capacity to form biofilm ( $p=0.017$ ) and a higher susceptibility to gentamycin and amoxicillin/clavulanate ( $\mathrm{p}=0.049$ both).

There were significant differences between biofilm-forming and non-biofilm-forming isolates concerning the iucA, iroN, and rmpA genes, with biofilm-forming strains lacking these genes ( $\mathrm{p}=0.02, \mathrm{p}=0.01$, and $\mathrm{p}<0.001$, respectively).
In the case of uropathogenic strains, we found a statistically significant association between the presence of the irp2, ybtS and fyuA genes and susceptibility to fosfomycin ( $\mathrm{p}=0.017, \mathrm{p}=$ 0.024 , and $p=0.024$, respectively). Similarly, we found significant associations between the presence of the uge gene and susceptibility to aztreonam, cefepime, ceftazidime, chloramphenicol, ciprofloxacin and trimethoprim-sulfamethoxazole $(\mathrm{p}=0.002, \mathrm{p}=$ $0.008, \mathrm{p}<0.001, \mathrm{p}=0.004, \mathrm{p}=0.018$ and $\mathrm{p}=0.043$, respectively). On the other hand, we found that the strains lacking the iucA gene were more susceptible to imipenem $(p<0.001)$ and chloramphenicol $(\mathrm{p}=0.013)$, whereas the strains lacking the iroN gene were more susceptible to piperacillin/tazobactam $(p=0.001)$.

Among the respiratory strains, we found a statistically significant association between the presence of the iucA gene and susceptibility to imipenem ( $p=0.036$ ), piperacillin/ tazobactam $(\mathrm{p}=0.034)$, amoxicillin/clavulanate $(\mathrm{p}=0.021)$, aztreonam $(p=0.006)$, cefepime $(p=0.036)$, ciprofloxacin $(\mathrm{p}=0.004)$ and trimethoprim-sulfamethoxazole $(\mathrm{p}=0.036)$. Similarly, we found a significant association between the presence of the $r m p A$ gene and susceptibility to piperacillin/ tazobactam $(\mathrm{p}=0.022)$, amoxicillin/clavulanate $(\mathrm{p}=0.025)$, aztreonam $(\mathrm{p}=0.031)$ and ciprofloxacin $(\mathrm{p}=0.022)$. The presence of the uge gene was correlated with susceptibility to aztreonam $(\mathrm{p}=0.005)$ and ciprofloxacin $(\mathrm{p}=0.012)$. However, strains lacking the $y b t S$, irp 2 or $f y u A$ genes were more susceptible to gentamicin ( $\mathrm{p}=0.047$ in all cases). Finally, strains carrying the $y b t S$ gene were more resistant to imipenem $(\mathrm{p}=0.039)$.

For the group of strains isolated from blood, we found a significant association between the presence of the uge gene and susceptibility to amoxicillin/clavulanate $(\mathrm{p}=0.001)$, aztreonam $(\mathrm{p}<0.001)$, cefepime $(\mathrm{p}<0.001)$, ceftazidime $(\mathrm{p}<0.001)$, chloramphenicol $(\mathrm{p}=0.024)$, ciprofloxacin $(\mathrm{p}<0.001)$, gentamicin $(p=0.003)$ and trimethoprim-sulfamethoxazole $(\mathrm{p}=0.012)$. Nevertheless, strains lacking the iucA or $y b t S$ genes were more susceptible to chloramphenicol $(p=0.024$ and $\mathrm{p}=0.016$ ). Similarly, strains lacking the $\operatorname{irp} 2$ gene were more susceptible to cefepime $(\mathrm{p}=0.044)$ and gentamicin $(\mathrm{p}=$ $0.044)$. Finally, strains carrying the $\operatorname{irp} 2$ gene were more resistant to aztreonam $(\mathrm{p}=0.027)$. 


\section{DISCUSSION}

$K$. pneumoniae causes a wide range of infections and uses different virulence factors to colonize and spread in the human body. The increasing antimicrobial resistance of this bacteria in recent years is of great concern to the scientific community.

In this study, we characterized a collection of 127 clinical $K$. pneumoniae strains isolated from urine, the respiratory tract, and blood and compared these strains according to antimicrobial resistance and the presence of a wide variety of virulence factors.

Overall, we found that more than $40 \%$ of isolates were classified as MDR and almost half of these were from urine. The data from the 2017 European Antimicrobial Resistance Surveillance Network report showed that at the European Union and the European economic area level, more than one third of $K$. pneumoniae isolates were resistant to at least one of the antimicrobial groups under regular surveillance. The report showed resistance percentages for fluoroquinolones (31.5\%), followed by third-generation cephalosporins (31.2\%), aminoglycosides (24.1\%), and carbapenems (7.2\%) (European Centre for Disease Prevention and Control (ECDC), 2018) similar to the general percentages observed in this study.

Urine isolates showed the highest overall antibiotic resistance (64.91\%), closely followed by strains isolated from blood $(63.64 \%)$ and the respiratory tract $(51.35 \%)$. Uropathogenic isolates made up the majority of the ESBL-producing strains. Compared to the multicenter study carried out for GarciaFernandez et al., our strains showed a higher prevalence of ESBL and lower carbapenemase production. This previous study reported a prevalence of $23.1 \%$ of ESBL-producing Klebsiella spp. and $20 \%$ of carbapenemase-producing Klebsiella (García-Fernández et al., 2019). This is of great concern because some antibiotic resistance genes are carried by mobile genetic elements that facilitate horizontal genetic exchange and promote the spread of antimicrobial resistance genes within and between species. Their characterization in future studies could help us to expand our research. On the other hand, multidrug resistance has been associated with increased health care costs, longer hospital stays, and high mortality rates. Moreover, nowadays there are few new alternatives for effective treatments.

Urine strains also showed the highest resistance rates to fosfomycin (24.56\%). Fosfomycin trometamol is one of the antibiotics proposed for treating uncomplicated UTIs, and its consumption has increased by $20.7 \%$ since 2012 in Spain, according to the Joint Inter-agency Antimicrobial Consumption and Resistance Analysis report (Alonso Herreras et al., 2018). Sorlozano et al. showed that ESBL-producing Klebsiella had low susceptibility rates to fosfomycin ranging between 40 and $78 \%$ (Sorlozano et al., 2014). Therefore, it is important to consider this antibiotic in the persistent monitoring and surveillance of antimicrobial resistance.

Regarding virulence, hypermucoviscous pathotypes, genes for adhesins, iron uptake and transport, toxins, and biofilm formation ability are some of the factors that $K$. pneumoniae employs in pathogenesis. Although many studies define hypervirulent $K$. pneumoniae (hvKp) as string test positive, it has been demonstrated that not all hvKp strains are hypermucoviscous (Fu et al., 2018). The study carried out by Russo et al. proposed the $\operatorname{rmp} A$ and $i u c A$ genes as biomarkers for distinguishing between hypervirulent (hvKp) and the classical (cKp) K. pneumoniae pathotypes (Russo et al., 2011). rmpA is a plasmid-located virulence factor gene that regulates the synthesis of capsular polysaccharides (Wang et al., 2020), and iucA is involved in the biosynthesis of the siderophore aerobactin (De Lorenzo and Neilands, 1986). These genes were significantly associated with the hvKp-phenotype in our study, in which seven of the $17(41.18 \%)$ hypermucoviscous strains carried both genes. On the other hand, two more strains were found, icuA+, rmpA+ and HMV-, five were classified as $i c u A+, r m p A-$ and HMV- and ten were classified as $i c u A-, r m p A$ - and $\mathrm{HMV}+$. This suggests that other regulatory mechanisms may be involved in the expression of the hypermucoviscous phenotype. Thus, considering both the string test and the hypervirulence-related genes iucA and $\operatorname{rmp} A, 24$ strains (18.9\%) were classified as hypervirulent in the present work. In terms of antimicrobial resistance, hvKp have been classified as susceptible or as ESBLproducer strains (Harada and Doi, 2018). We found 13 strains classified as susceptible and nine as ESBL-producers, similar to the studies previously described. Of the other two strains with an $i c u A-, r m p A-$ and HMV+ phenotype, one was classified as resistant to fosfomycin and the other as resistant to more than three categories of antibiotics.

Other hypervirulence-associated genes, such as iroBCDN, iutA, iuacABCD, ybt and the clb locus, may be encoded in the chromosome as part of integrative conjugative elements (Arato et al., 2021). The hypervirulent phenotype confers important properties to bacteria for pathogenesis, such as capsule polysaccharide production, phagocytosis resistance, dissemination and systemic infection in the host (Xu et al., 2021). Early identification of hvKp strains would allow better understanding of the infection and the implementation of more effective antimicrobial treatment. Likewise, it is mandatory to avoid the possible dissemination of these pathotypes by implementing barriers between patients, with precautions based on virulence likely being as important as those based on antimicrobial resistance.

Among siderophores, enterobactin (encoded by ent genes), aerobactin (encoded by iuc genes), salmochelin (encoded by iro genes), and yersiniabactin (encoded by $y b t$ genes, regulated by irp genes, and the receptor is encoded by the fyu $A$ gene) are the most representative in $K$. pneumoniae. Siderophores are indispensable to obtain the iron necessary for the growth and replication of bacteria. They scavenge iron from host transport proteins. Enterobactin is produced by all $K$. pneumoniae, but its mechanism may be disabled by human lipocalin-2 (Lcn2), which has an affinity for enterobactin and elicits an inflammatory response. In contrast, yersiniabactin eludes binding to $\mathrm{Lcn} 2$, thereby avoiding the inflammatory response and promoting bacterial growth (Lam et al., 2018). As expected, we found the entB gene in all the strains tested in agreement with another study (Fatima et al., 2021). Besides, the higher prevalence of the siderophore-encoded $y b t S$ and $f y u A$ genes in the respiratory tract isolates compared to the urinary 
and blood strains confirmed that yersiniabactin is an important virulence factor for the pulmonary infection, as reported by previous studies (Yan et al., 2016).

The capsule-associated genes wabG, (encoding capsule), uge (encoding capsule lipoprotein) and $y c f M$ (promoting external membrane protein) are highly involved in virulence by promoting infection through resistance to phagocytosis. We observed the presence of the uge (uridine diphosphate galacturonate- 4 epimerase) gene in the majority of the isolates from urine, showing a statistically significant difference compared to the other clinical isolates. It has been demonstrated that K. pneumoniae strains lacking the uge gene are less virulent and less capable of causing UTI, pneumonia or sepsis. Mutations in the uge gene reduce the colonization ability of $K$. pneumoniae in experimentally induced urinary infections (Regue et al., 2004; Candan and Aksöz, 2015; Remya et al., 2019). However, the occurrence of the uge gene in K. pneumoniae has varied widely in different studies. Remya et al. found a prevalence of $48.6 \%$ harboring the uge gene. Its distribution in different clinical specimens was blood (50\%), urine (48.8\%), exudates (48.4\%), and respiratory secretions (47.3\%). El FertasAissani et al. observed the presence of the uge gene in $84.6 \%$ of urinary tract strains and all the blood and respiratory isolates (El Fertas-Aissani et al., 2013). Although the uge gene has previously been described as essential for $K$. pneumoniae virulence, future research may provide further evidence for its specific association with UTIs.

Among the toxins tested, two of the strains were positive for the $c l b A$ and $c l b Q$ genes, two of the genes encoding colibactin. Colibactin is a recently described toxin that has been associated with colorectal cancer in humans because it causes DNA damage in host cells. Colibactin has been found in E. coli, but its occurrence in other species is steadily increasing. Acquisition of the pks locus, which synthesizes this toxin, has been linked to intestinal colonization and mucosal invasion by K. pneumoniae (Strakova et al., 2021).

Interestingly, one of the strains isolated from the respiratory tract was positive for all the virulence genes tested. Two other strains were positive for almost all the virulence genes except those encoding colibactin. These three strains were susceptible to all antimicrobial agents, and were classified as serotype K1, nonbiofilm-forming, or weak biofilm-forming strains and showed high serum resistance, which protects the bacteria from phagocytosis (Hennequin and Robin, 2016). Although more research is needed on the influence of the acquisition of antibiotic resistance mechanisms by the bacteria on fitness cost and the relation with virulence, we found a scenario in which the greater the virulence, the lesser the antimicrobial resistance. Indeed, we found a correlation between the presence of some virulence factor genes and susceptibility to most of the antibiotics tested.

The genes encoding ESBL are carried by plasmids that may also harbor other genes, including some virulence factor genes. In previous studies, three virulence factors (adhesin CF29K, aerobactin or mucoid phenotype) were found in ESBLproducing $K$. pneumoniae strains. (Hennequin and Robin, 2016). In our study, we found the same association between $f y u A$, irp2 or $y b t S$ genes and ESBL-producing strains. The $y b t$ carried by the $\mathrm{FIB}_{\mathrm{K}}$ plasmid is a novel mechanism for $y b t$ mobilization, Currently, many $\mathrm{FIB}_{\mathrm{K}}$ plasmids also contain antimicrobial resistance transposons, which may indicate the coexistence of virulence and resistance genes in the same mobile genetic element (Lam et al., 2018).

Regarding biofilm, almost half of biofilm-forming strains, including weak, moderate and strong, were isolated from urine and the difference from the other clinical sources was statistically significant. K. pneumoniae has been identified as the second most prevalent pathogen in UTIs (Liu et al., 2020), and the ability to form biofilms on indwelling urethral catheters is one of the most common conditions, affecting thousands of people worldwide each year. Recurrence of catheter urinary tract infections after antibiotic treatment could be caused by re-colonization by bacteria that survived in the biofilm (Stahlhut et al., 2012). Besides, biofilms present higher resistance to phagocytosis and higher antimicrobial resistance compared to planktonic bacteria (Trautner and Darouiche, 2004). Nonetheless, truly effective strategies to prevent or counteract biofilm-related infections remain elusive.

\section{CONCLUSION}

We found that isolates from urine were more resistant to antimicrobials and were more ESBL-producers, and more biofilm-formers compared to respiratory and blood strains. Our study also correlated the presence of the uge gene with UTIs and the presence of yersiniabactin with respiratory tract infections. In addition, although we found an inverse relation between antimicrobial resistance and virulence, the acquisition of mobile genetic elements could promote not only the spread of antimicrobial resistance genes but also that of virulence genes that evolve toward pathotypes considered to be more virulent. The increasing coexistence of these two conditions is of particular concern as it can lead to untreatable and invasive $K$. pneumoniae infections. Active surveillance not only for antimicrobial resistance but also for virulence determinants is imperative to avoid the transmission and spread of hypervirulent or extensively resistant strains.

\section{DATA AVAILABILITY STATEMENT}

The original contributions presented in the study are included in the article/Supplementary Material. Further inquiries can be directed to the corresponding author.

\section{AUTHOR CONTRIBUTIONS}

Conceptualization, SS and VB. Methodology, VB, YG, MT, RO, and CR. Statistical analysis, VB. Writing-original draft preparation, VB and SS. Writing-review and editing, VB, CR, 
YG, and SS. All authors contributed to the article and approved the submitted version.

\section{FUNDING}

This work was funded by Planes Nacionales de I+D+iI20082011/2013-2016 and Instituto de Salud Carlos III (PI19/00478), Subdirección General de Redes y Centros de Investigación Cooperativa, Ministerio de Economía y Competitividad, Spanish Network for Research in Infectious Diseases (REIPI RD12/0015/0013 and REIPI RD16/0016/0010) co-financed by European Development Regional Fund "A way to achieve

\section{REFERENCES}

Alonso Herreras, M., Aracil García, B., Saiz,, Badiola, I., Campos Marqués, J., Durán Ferrer, M., et al. (2018). Informe JIACRA España. Primer Análisis Integrado Del Consumo De Antibióticos Y Su Relación Con La Aparición De Resistencia. AEMPS. Plan Nac. Resist. Antibióticos, 1-165.

Arato, V., Raso, M. M., Gasperini, G., Scorza, F. B., and Micoli, F. (2021). Prophylaxis and Treatment Against Klebsiella Pneumoniae: Current Insights on This Emerging Anti-Microbial Resistant Global Threat. Int. J. Mol. Sci. 22, 4042-4062. doi: 10.3390/ijms22084042

Candan, E. D., and Aksöz, N. (2015). Klebsiella Pneumoniae: Characteristics of Carbapenem Resistance and Virulence Factors. Acta Biochim. Pol. 62, 867-874. doi: 10.18388/abp.2015_1148

CLSI (2020). CLSI. Performance Standards for Antimicrobial Susceptibility Testing. 30th Edition Vol. CLSI Supplement M100 (Wayne, PA, USA: Clinical and Laboratory Standard Institute). Available at: https://clsi.org/standards/ products/microbiology/documents/m100/.

Compain, F., Babosan, A., Brisse, S., Genel, N., Audo, J., Ailloud, F., et al. (2014). Multiplex PCR for Detection of Seven Virulence Factors and K1/K2 Capsular Serotypes of Klebsiella Pneumoniae. J. Clin. Microbiol. 52, 4377-4380. doi: 10.1128/JCM.02316-14

De Lorenzo, V., and Neilands, J. B. (1986). Characterization of iucA and iucC Genes of the Aerobactin System of Plasmid ColV-K30 in Escherichia Coli. J. Bacteriol. 167, 350-355. doi: 10.1128/jb.167.1.350-355.1986

Dybowska-Sarapuk, Ł., Kotela, A., Krzemiński, J., Wróblewska, M., Marchel, H., Romaniec, M., et al. (2017). Graphene Nanolayers as a New Method for Bacterial Biofilm Prevention: Preliminary Results. J. AOAC Int. 100, 900-904. doi: 10.5740/jaoacint.17-0164

El Fertas-Aissani, R., Messai, Y., Alouache, S., and Bakour, R. (2013). Virulence Profiles and Antibiotic Susceptibility Patterns of Klebsiella Pneumoniae Strains Isolated From Different Clinical Specimens. Pathol. Biol. 61, 209-216. doi: $10.1016 /$ j.patbio.2012.10.004

European Centre for Disease Prevention and Control (ECDC) (2018). "ECDC: SURVEILLANCE REPORT," in Surveillance of Antimicrobial Resistance in Europe 2017(Stockholm). Available at: https://ecdc.europa.eu/en/publicationsdata/antimicrobial-resistance-surveillance-europe-2016.

Fang, C. T., Chuang, Y. P., Shun, C. T., Chang, S. C., and Wang, J. T. (2004). A Novel Virulence Gene in Klebsiella Pneumoniae Strains Causing Primary Liver Abscess and Septic Metastatic Complications. J. Exp. Med. 199, 697-705. doi: 10.1084/jem.20030857

Fatima, S., Liaqat, F., Akbar, A., Sahfee, M., Samad, A., Anwar, M., et al. (2021). Virulent and Multidrug-Resistant Klebsiella Pneumoniae From Clinical Samples in Balochistan. Int. Wound J. 18, 1-9. doi: 10.1111/iwj.13550

Foxman, B. (2010). The Epidemiology of Urinary Tract Infection. Nat. Rev. Urol. 7, 653-660. doi: 10.1038/nrurol.2010.190

Fu, L., Huang, M., Zhang, X. Z., Yang, X. Y., Liu, Y., Zhang, L. H., et al. (2018). Frequency of Virulence Factors in High Biofilm Formation blaKPC-2 Producing Klebsiella Pneumoniae Strains From Hospitals. Microb. Pathog. 116, 168-172. doi: 10.1016/j.micpath.2018.01.030

García-Fernández, S., García-Castillo, M., Bou, G., Calvo, J., Cercenado, E., Delgado, M., et al. (2019). Activity of Ceftolozane/Tazobactam Against
Europe" and operative program Intelligent Growth 2014-2020. ISGlobal is a CERCA center from the Generalitat of Catalunya and a Severo Ochoa Center (Spanish Ministry of Science, Innovations, and Universities). VB has a grant from Ministerio de Ciencia, Tecnología e Innovación (Colombia).

\section{SUPPLEMENTARY MATERIAL}

The Supplementary Material for this article can be found online at: https://www.frontiersin.org/articles/10.3389/fcimb.2021. 738223/full\#supplementary-material

Pseudomonas Aeruginosa and Enterobacterales Isolates Recovered From Intensive Care Unit Patients in Spain: The SUPERIOR Multicentre Study. Int. J. Antimicrob. Agents 53, 682-688. doi: 10.1016/j.ijantimicag.2019.02.004 Garza-Ramos, U., Barrios-Camacho, H., Moreno-Domínguez, S., Toribio-Jiménez, J., Jardón-Pineda, D., Cuevas-Peña, J., et al. (2018). Phenotypic and Molecular Characterization of Klebsiella Spp. Isolates Causing Community-Acquired Infections. New Microbes New Infect. 23, 17-27. doi: 10.1016/j.nmni.2018.02.002

Guerra, B., Junker, E., Miko, A., Helmuth, R., and Mendoza, M. C. (2004). Characterization and Localization of Drug Resistance. Microb. Drug Resist. 10, 83-91. doi: 10.1089/1076629041310136

Guo, Y., Zhou, H., Qin, L., Pang, Z., Qin, T., Ren, H., et al. (2016). Frequency, Antimicrobial Resistance and Genetic Diversity of. Klebsiella pneumoniae Food Samples. PloS One 11.

Harada, S., and Doi, Y. (2018). Hypervirulent Klebsiella Pneumoniae: A Call for Consensus Definition and International Collaboration. J. Clin. Microbiol. 56, 1-4. doi: 10.1128/JCM.00959-18

Hennequin, C., and Robin, F. (2016). Correlation Between Antimicrobial Resistance and Virulence in Klebsiella Pneumoniae. Eur. J. Clin. Microbiol. Infect. Dis. 35, 333-341. doi: 10.1007/s10096-015-2559-7

Jian-li, W., Yuan-yuan, S., Shou-yu, G., Fei-fei, D., Jia-yu, Y., Xue-hua, W., et al. (2017). Serotype and Virulence Genes of Klebsiella Pneumoniae Isolated From Mink and Its Pathogenesis in Mice and Mink. Sci. Rep. 7, 1-7. doi: 10.1038/s41598-017-17681-8

Lam, M. M. C., Wick, R. R., Wyres, K. L., Gorrie, C. L., Judd, L. M., Jenney, A. W. J., et al. (2018). Genetic Diversity, Mobilisation and Spread of the Yersiniabactin- Encoding Mobile Element ICE Kp in Klebsiella Pneumoniae Populations. Microb. Genomics 4, e000196. doi: 10.1099/mgen.0.000196

Lin, J. C., Koh, T. H., Lee, N., Fung, C. P., Chang, F. Y., Tsai, Y. K., et al. (2014). Genotypes and Virulence in Serotype K2 Klebsiella Pneumoniae From Liver Abscess and Non-Infectious Carriers in Hong Kong, Singapore and Taiwan. Gut Pathog. 6, 1-7. doi: 10.1186/1757-4749-6-21

Liu, X., Sai, F., Li, L., Zhu, C., and Huang, H. (2020). Clinical Characteristics and Risk Factors of Catheter-Associated Urinary Tract Infections Caused by. Klebsiella pneumoniae. Ann. Cardiothorac. Surg. 9, 2668-2677. doi: 10.21037/apm-20-1052

Magiorakos, A. P., Srinivasan, A., Carey, R. B., Carmeli, Y., Falagas, M. E., Giske, C. G., et al. (2012). Multidrug-Resistant, Extensively Drug-Resistant and Pandrug-Resistant Bacteria: An International Expert Proposal for Interim Standard Definitions for Acquired Resistance. Clin. Microbiol. Infect. 18, 268-281. doi: 10.1111/j.1469-0691.2011.03570.x

Monstein, H. J., Östholm-Balkhed, Å., Nilsson, M. V., Nilsson, M., Dornbusch, K., and Nilsson, L. E. (2007). Multiplex PCR Amplification Assay for the Detection of blaSHV, blaTEM and blaCTX-M Genes in Enterobacteriaceae. Apmis 115, 1400-1408. doi: 10.1111/j.1600-0463.2007.00722.x

Morgan, R. N., Saleh, S. E., Farrag, H. A., and Aboulwafa, M. M. (2019). Prevalence and Pathologic Effects of Colibactin and Cytotoxic Necrotizing Factor-1 (Cnf 1) in Escherichia Coli: Experimental and Bioinformatics Analyses. Gut Pathog. 11, 1-18. doi: 10.1186/s13099-019-0304-y

O'Toole, G., and Kolter, R. (1998). Initiation of Biofilm Formation in Pseudomonas Fluorescens WCS365. Mol. Microbiol. 28, 449-461. doi: 10.1046/j.1365-2958.1998.00797.x

Park, C. H., Robicsek, A., Jacoby, G. A., Sahm, D., and Hooper, D. C. (2006). Prevalence in the United States of $\mathrm{Aac}\left(6^{\prime}\right)-\mathrm{Ib}-\mathrm{Cr}$ Encoding a Ciprofloxacin 
Modifying Enzyme. Antimicrob. Agents Chemother. 50, 3953-3955. doi: 10.1128/AAC.00915-06

Podschun, R., Sievers, D., Fischer, A., and Ullmann, U. (2016). Serotypes, Hemagglutinins, Siderophore Synthesis, and Serum Resistance of Klebsiella Isolates Causing Human Urinary Tract Infections Vol. 168 (Oxford University Press Stable URL), 1415-1421.

Podschun, R., and Ullmann, U. (1998). Klebsiella Spp. As Nosocomial Pathogens: Epidemiology, Taxonomy, Typing Methods, and Pathogenicity Factors. Clin. Microbiol. Rev. 11, 589-603. doi: 10.1128/cmr.11.4.589

Queenan, A. M., and Bush, K. (2007). Carbapenemases: The Versatile $\beta$ Lactamases. Clin. Microbiol. Rev. 20, 440-458. doi: 10.1128/CMR.00001-07

Regué, M., Hita, B., Piqué, N., Izquierdo, L., Merino, S., Fresno, S., et al. (2004). A Gene, Uge, Is Essential for Klebsiella Pneumoniae Virulence. Infect. Immun. 72, 54-61. doi: 10.1128/IAI.72.1.54-61.2004

Remya, P. A., Shanthi, M., and Sekar, U. (2019). Characterisation of Virulence Genes Associated With Pathogenicity in Klebsiella Pneumoniae. Indian J. Med. Microbiol. 37, 210-218. doi: 10.4103/ijmm.IJMM_19_157

Robicsek, A., Strahilevitz, J., Jacoby, G. A., Macielag, M., Abbanat, D., Chi, H. P., et al. (2006). Fluoroquinolone-Modifying Enzyme: A New Adaptation of a Common Aminoglycoside Acetyltransferase. Nat. Med. 12, 83-88. doi: $10.1038 / \mathrm{nm} 1347$

Russo, T. A., Shon, A. S., Beanan, J. M., Olson, R., MacDonald, U., Pomakov, A. O., et al. (2011). Hypervirulent K. Pneumoniae Secretes More and More Active Iron-Acquisition Molecules Than "Classical" K. Pneumoniae Thereby Enhancing its Virulence. PloS One 6. doi: 10.1371/journal.pone.0026734

Sorlozano, A., Jimenez-Pacheco, A., De Dios Luna Del Castillo, J., Sampedro, A., Martinez-Brocal, A., Miranda-Casas, C., et al. (2014). Evolution of the Resistance to Antibiotics of Bacteria Involved in Urinary Tract Infections: A 7-Year Surveillance Study. Am. J. Infect. Control 42, 1033-1038. doi: 10.1016/ j.ajic.2014.06.013

Stahlhut, S. G., Struve, C., Krogfelt, K. A., and Reisner, A. (2012). Biofilm Formation of Klebsiella Pneumoniae on Urethral Catheters Requires Either Type 1 or Type 3 Fimbriae. FEMS Immunol. Med. Microbiol. 65, 350-359. doi: 10.1111/j.1574-695X.2012.00965.x

Stepanović, S., Vuković, D., Hola, V., Di Bonaventura, G., Djukić, S., Ćirković, I., et al. (2007). Quantification of Biofilm in Microtiter Plates: Overview of Testing Conditions and Practical Recommendations for Assessment of Biofilm Production by. staphylococci. Apmis 115, 891-899. doi: 10.1111/j.16000463.2007.apm_630.x

Strakova, N., Korena, K., and Karpiskova, R. (2021). Klebsiella Pneumoniae Producing Bacterial Toxin Colibactin as a Risk of Colorectal Cancer Development - A Systematic Review. Toxicon 197, 126-135. doi: 10.1016/ j.toxicon.2021.04.007
Trautner, B. W., and Darouiche, R. (2004). Role of Biofilm in Catheter-Associated Urinary Tract Infection. Am. J. Infect. Control 32, 177-183. doi: 10.1016/ j.ajic.2003.08.005

Wang, G., Zhao, G., Chao, X., Xie, L., and Wang, H. (2020). The Characteristic of Virulence, Biofilm and Antibiotic Resistance of. Klebsiella pneumoniae. Int. J. Environ. Res. Public Health 17, 1-17. doi: 10.3390/ijerph17176278

Wiegand, I., Geiss, H. K., Mack, D., Stu, E., Seifert, H.Icrobiol, J. C. L. I. N. M. (2007). Detection of Extended-Spectrum Beta-Lactamases Among Enterobacteriaceae by Use of Semiautomated Microbiology Systems and Manual Detection Procedures. J. Clin. Microbiol. 45, 1167-1174. doi: 10.1128/ JCM.01988-06

Woodford, N., Fagan, E. J., and Ellington, M. J. (2006). Multiplex PCR for Rapid Detection of Genes Encoding CTX-M Extended-Spectrum $\beta$-Lactamases [4]. J. Antimicrob. Chemother. 57, 154-155. doi: 10.1093/jac/dki412

World Health Organization (2017). Global Priority List of Antibiotic-Resistant Bacteria to Guide Research, Discovery, and Development of New Antibiotics (WHO Press), 1-7.

Xu, Q., Yang, X., Wai, E., Chan, C., and Chen, S. (2021). The Hypermucoviscosity of Hypervirulent K . Pneumoniae Confers the Ability to Evade NeutrophilMediated Phagocytosis. Virulence 12, 2050-2059. doi: 10.1080/21505594. 2021.1960101

Yan, Q., Zhou, M., Zou, M., and Liu, W. e. (2016). Hypervirulent Klebsiella Pneumoniae Induced Ventilator-Associated Pneumonia in Mechanically Ventilated Patients in China. Eur. J. Clin. Microbiol. Infect. Dis. 35, 387-396. doi: 10.1007/s10096-015-2551-2

Conflict of Interest: The authors declare that the research was conducted in the absence of any commercial or financial relationships that could be construed as a potential conflict of interest.

Publisher's Note: All claims expressed in this article are solely those of the authors and do not necessarily represent those of their affiliated organizations, or those of the publisher, the editors and the reviewers. Any product that may be evaluated in this article, or claim that may be made by its manufacturer, is not guaranteed or endorsed by the publisher.

Copyright (๑ 2021 Ballén, Gabasa, Ratia, Ortega, Tejero and Soto. This is an openaccess article distributed under the terms of the Creative Commons Attribution License (CC BY). The use, distribution or reproduction in other forums is permitted, provided the original author(s) and the copyright owner(s) are credited and that the original publication in this journal is cited, in accordance with accepted academic practice. No use, distribution or reproduction is permitted which does not comply with these terms. 